\title{
Research on a Device about Zero Line Break
}

\section{Zheng Fuchun, Chang Jianing, Zong Fei, Zhang Hao, Li Shengjun, Zhou Debo*, Tao Lei, Tang Shijun, Zhou Liangjun, Zhou Zhiwei, Chen Shili, Tan Xianghua, Liu Yi}

Changshou Electric Power Company of State Grid, Changshou, China

\section{Email address:}

1014213363@qq.com (Zheng Fuchun),79133833@qq.com (Chang Jianing),1328506378@qq.com (Zhou Debo)

*Corresponding author

\section{To cite this article:}

Zheng Fuchun, Chang Jianing, Zong Fei, Zhang Hao, Li Shengjun, Zhou Debo, Tao Lei, Tang Shijun, Zhou Liangjun, Zhou Zhiwei, Chen Shili, Tan Xianghua, Liu Yi. Research on a Device about Zero Line Break. Science Discovery. Vol. 7, No. 5, 2019, pp. 337-340. doi: $10.11648 /$ j.sd.20190705.23

Received: November 1, 2019; Accepted: November 21, 2019; Published: December 3, 2019

\begin{abstract}
In the three-phase four wires system, the neutral line is uncharged under normal circumstances, because any point of the neutral line and the earth are of the same potential. However, when the zero line is disconnected, the neutral point on the load side will generate displacement voltage. At this time, if people touch the broken zero line, the current will form a path from the phase line load zero line human body zero point phase line, which is extremely dangerous and the rise of zero line voltage will also cause damage to electrical equipment. In order to solve the harm of zero line fault, this paper puts forward a zero line fault early warning device, which includes voltage acquisition, logic judgment ,attention device and telecommunication equipment, This device can effectively detect the fault points with poor zero line contact, alarm the fault, prompt the staff to check, reduce economic losses and casualties, and improve the safety of power use.
\end{abstract}

Keywords: Zero Line, Disconnection, Early Warning Device

\section{一种关于零线断线装置的研究}

郑富春, 常家宁, 纵飞, 张浩, 李胜军, 周德波 ${ }^{*}$ 陶否, 唐世军, 周良军, 周志伟, 陈世李, 谈祥华, 刘义

国网重庆市电力公司长寿供电分公司, 长寿, 中国

\section{邮箱}

1014213363@qq.com(郑富春)，79133833@qq.com（常家宁），1328506378@qq.com（周德波）

摘要: 在三相四线制中性点直接接地的线路中, 正常情况下零线是不带电的, 这是因为正常时零线上任一点和大地都 是同电位的缘故。但是在零线断线后, 负荷侧的中性点会产生位移电压, 此时若人碰到断落的零线, 电流将由相线一 负载一零线一人体一零点一相线形成通路, 这是极为危险的,并且零线电压的抬升还将造成用电设备的损坏。为解决低 压配电端因零线故障而带来的相关危害, 本文根据低压端零线实际运行情况研制了一种集电压采样、信号分析、现场 与远程预警为一体的零线故障预警装置, 初步测试表明, 本装置能够有效检测出零线接触不良的故障点, 并进行故障 报警, 以供工作人员进行排查参考, 减少经济损失和人员伤亡, 提高用电安全性。

关键词: 零线, 断线, 预警装置 


\section{1. 引言}

在三相四线低压配电网中, 经常会由于零线电流过大 或接头接触不良等原因造成零线接触电阻增加, 引起负荷 中性点电位偏移, 电压升高, 严重时可使某一相或两相的 相电压升高至 $300 \mathrm{~V}$ 以上, 给用户端用电设备造成很大威胁, 经常因此类故障烧毁家用电器 [1-5]。据长寿统计, 2012-2017年发生家电烧毁次数高达128次,其中发生因零 线接触不良引起的家电烧毁事件 102 件，占比高达 $80 \%$; 赔 偿金额为 94.9596 万元,其中因零线接触不良引起的家电烧 毁赔偿 72.1525 万元, 占比高达 $76 \%$ 。由此可见, 零线断线 已成为低压配电网主要电力事故 ${ }^{[6-8]}$ 。基层班组为索赔、 定损、维修、付款等事宜付出大量的人力, 其中, 零线与 零排的接触不良, 造成温度升高零线烧断, 是造成零线接 触不良的主要原因[9-15]。因此亟需研制一种低压零线接 触不良预警装置。
本文旨在针对现有低压配网中存在的零线断线故障 问题, 特别创新地研制了一种零线故障预警装置, 能够有 效检测出零线接触不良故障点，并进行及时预警，供检修 人员提前查看, 以减小零线断线事故所带来的设备与人员 安全问题。

\section{2. 方法}

为了达到上述目的, 本文研制了一种零线故障预警装 置, 包括电压采集模块, 包括零线引前电压采样电路和若 干条零线引后电压采样电路, 用以采集所需电压信号。A/D 转换模块, 用以将所采集的模拟信号转换为数字信号; 逻 辑判断模块, 用以对采集信号进行逻辑判断; 预警模块, 用以故障预警处理。

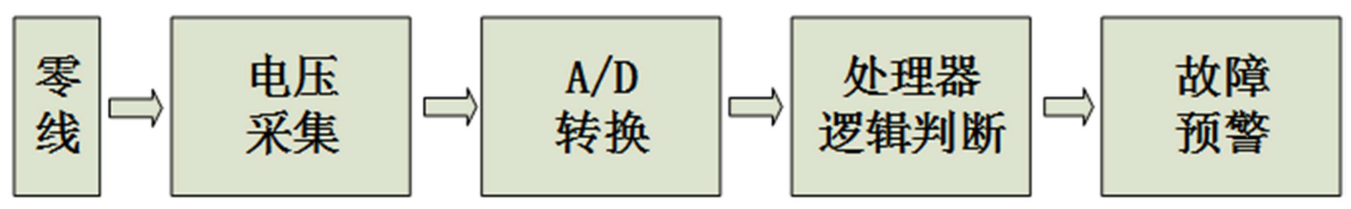

图1 低压零线接触不良预警方案。

工作过程中, 电压采集模块采集零线入线的电压信号 和所有零线出线的电压信号, 然后将信号传递给 $\mathrm{A} / \mathrm{D}$ 转换 器, 以便将电压采集模块的电压信号转换为与 CPU控制器 相匹配的数字信号。CPU控制器用于分别对比所有零线出 线与零线入线的电压是否有差值, 其差值是否超过允许范 围。若电压差值的超过允许范围, 则CPU控制器向报警输 出系统发送报警信号。详细接线图见图2所示。

图2中 (1)为零线入线, (2)为零排, (3)为零线出线, (4) 为零线电压信号采样及处理装置, (5)为预警终端。工作过 程中, (4)通过分别对各条零线出线与零线入线两端电压进 行采集, 以出线 1 为例, 若零线出线 1 与零排接触不良, 势 必导致 $\mathrm{A} 、 \mathrm{~B}$ 两端电压抬升, 当电压抬升超过一定范围后 (超出预先设定的电压阈值, 该阈值设置大于正常工作电 压, 但低于用电设备安全电压允许范围）, 视为存在接触 不良故障, (4)便判定出线1存在接触不良的问题，向(5)发
出故障信号, 并给出对应故障位置1, 由(5)做出故障预警。 实际过程中, 若所有出线所采集的电压信号均超过阈值, 需考虑入线与零排接触不良，入线故障预警。

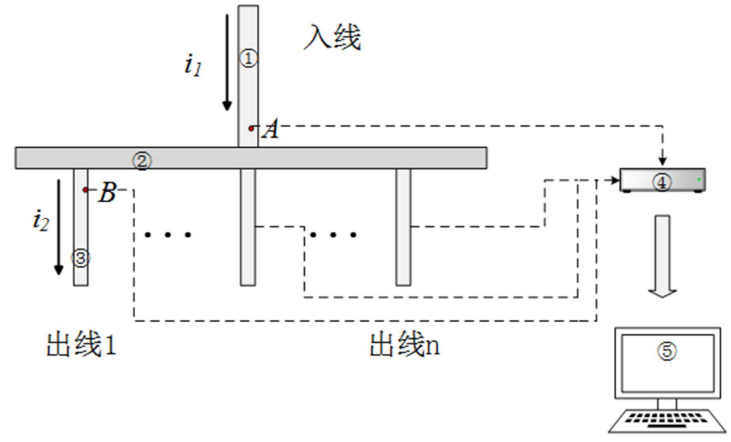

图2 低压零线接触不良预警装置详细接线图。

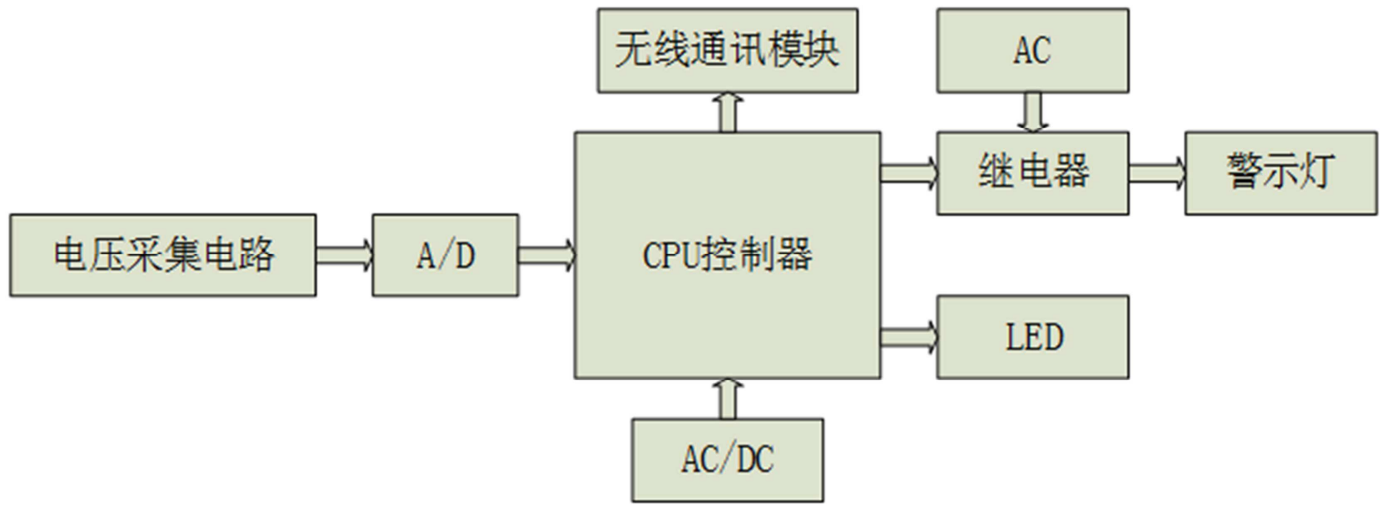




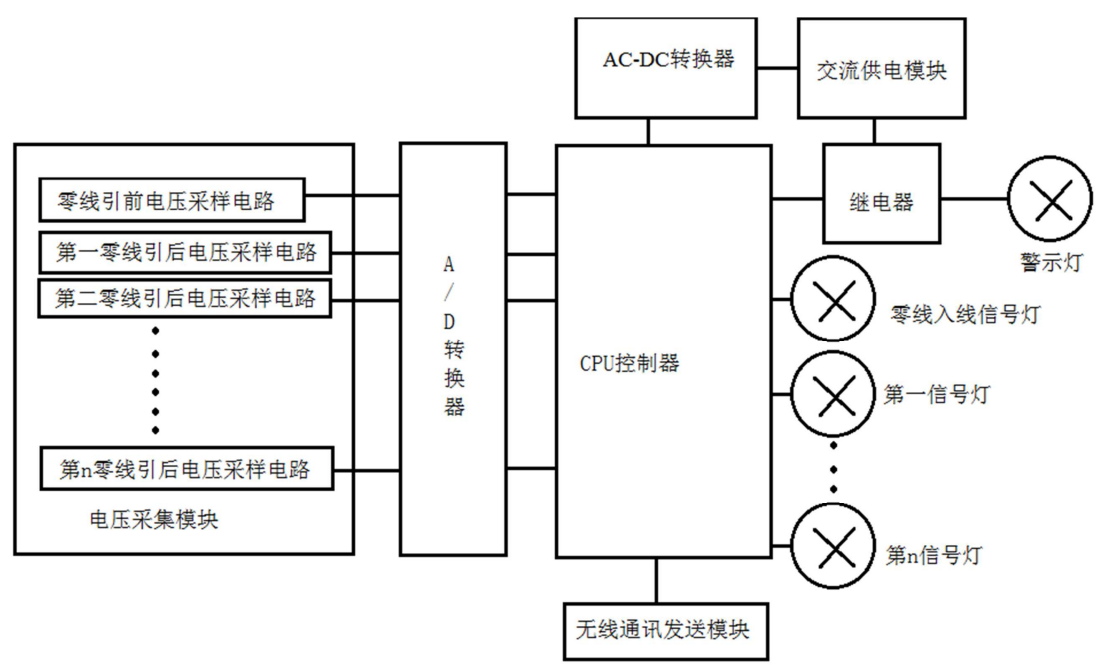

(b)

图3 零线接触不良预警装置结构示意图。

由于实际工作环境条件复杂, 所采集的信号需经过一 定处理后才能被用以分析, 因此需要设计对应的电压采样 电路。电压采样电路如图4所示。通过第一电阻 $\mathrm{R} 1$ 和第一 电容 $\mathrm{C} 1$ 组成 $\mathrm{RC}$ 滤波电路, 滤除电路中的谐波干扰, 减小 相位误差。第一运放U1作为电压跟随器, 提高电路的输入
阻抗, 通过第二运放U2进行过零比较, 通过第一二极管 D1和第二二极管 $\mathrm{D} 2$ 形成上拉钳位电路, 防止信号异常导 致元件损坏。通过第二电阻 $R 2$ 和第十一电阻R11抑制干扰 和信号的振荡。

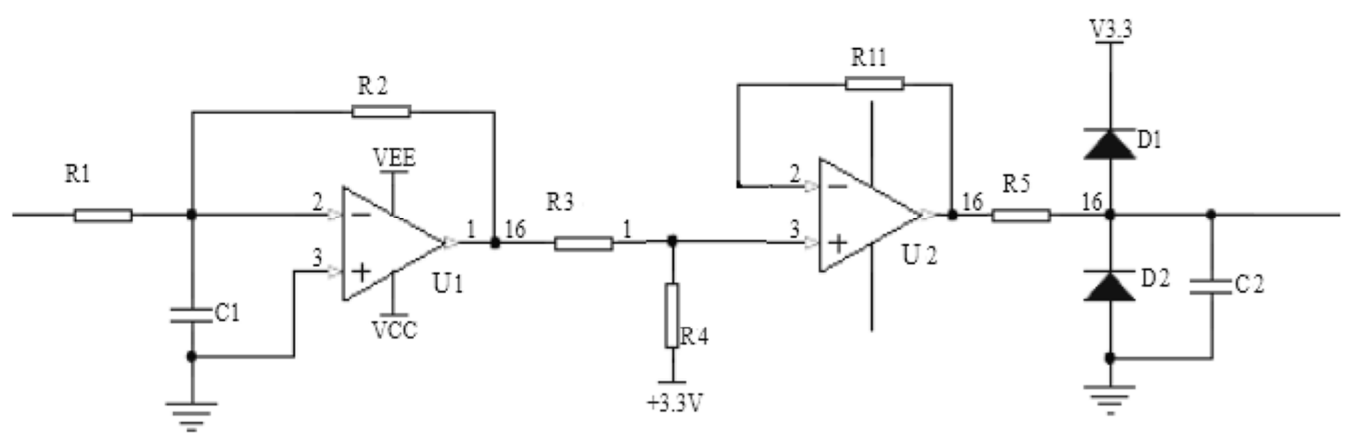

图4 电压采样电路。

经 $\mathrm{A} / \mathrm{D}$ 转换电路转换为数字信号后传递给 $\mathrm{CPU}$ 控制器 判断处理, 判定电压是否超出阈值, 并输出对应信号。电 压超出阈值后CPU控制器输出信号可控制两类预警终端 响应, 1 是现场预警:CPU控制器输出控制信号, 控制继电 器导通, 进而控制现场警示灯工作, 同时控制对应的LED 指示故障位置 (如1 号出线发生故障, 则装置上对应 1 号的 LED亮）。2是远程通讯: 通过无线通讯模块, 将故障信 息传至控制中心，供控制中心人员查看。

整套装置中继电器采用 $\mathrm{AC}$ 电源供电, $\mathrm{CPU}$ 控制器及 其余模块电路经 $\mathrm{AC} / \mathrm{DC}$ 转换后, 采用直流供电。

由此, 根据上述设计, 该装置能够分别对多条零线出 线进行单独采样分析, 并通过现场警示灯提示当地用户, 同时利用无线通信模块直接发送故障预警信息给相关检 修人员，便于检修人员及时处理。

\section{3. 初步验证}

为验证该装置的可行性, 将装置按要求接入配电箱
内, 通过改变配电箱内零线与零排接触状况来模拟零线 接线故障程度, 将如表 1 所示。

表1 四种接触状态的行程描述。

\begin{tabular}{lll}
\hline 序号 & 接触状态 & 接触状态现场图 \\
$\mathrm{A}$ & 完全接触状态 & \\
$\mathrm{B}$ & $+1 \mathrm{~mm}$ 接触状态 \\
$\mathrm{C}$ & $+2 \mathrm{~mm}$ 接触状态 \\
$\mathrm{D}$ & $+4 \mathrm{~mm}$ 接触状态 & \\
\hline
\end{tabular}

根据不同状况下实测电压设定装置预警阈值为 $30 \mathrm{~V}$, 并依次接入 8 个输入端, 对每个输入端进行测试, 试验验 证情况如表2所示。 
表2 目标效果检查表。

\begin{tabular}{lllll}
\hline 序号 & 试验电压值 $(\mathbf{V})$ & 电压越限阀值 $(\mathbf{V})$ & 报警状况 & 是否成功 \\
\hline $\mathrm{A}$ & $0-29$ & 30 & 否 & 是 \\
$\mathrm{B}$ & $30-49$ & 30 & 是 & 是 \\
$\mathrm{C}$ & $50-69$ & 50 & 是 & 是 \\
$\mathrm{D}$ & $70-80$ & 70 & 是 & 是 \\
\hline
\end{tabular}

根据初步验证结果, 所研制的零线故障预警装置能够 有效的对零线故障进行检测并作出预警，且能够准确提示 故障所处出现端口, 为检修人员提供排查参考, 减小了检 修排查工作量。

\section{4. 结论}

针对低压配网中所存在的零线故障问题, 本文研制了 一种零线故障预警装置, 主要包括电压采集模块、 $\mathrm{A} / \mathrm{D}$ 转 换模块、逻辑判断模块以及预警模块。通过初步验证, 该 装置能够有效检测出零线接触不良的故障点, 并进行故障 报警, 提示工作人员进行排查, 减少经济损失和人员伤亡, 提高用电安全性。

由于初期实验在认为设置电压以及少量样本的实际 运行工况下完成, 还不能完全验证该装置在实际运行过程 中的灵敏度及可靠性, 未来将在更多的配电端安装该装置, 以验证其在长期运行下的可行性, 为该装置的优化以及推 广提供可靠数据依据。

\section{参考文献}

[1] 朱则刚. 配电线路中性线断线故障的规范 [J]. 农村运维人 员， 2015，03:25。

[2] 潘群英, 张延志, 杨长春, 王兆新. 照明线路中性线断线 的危害与防范治理 [J]. 内江科技, 2013,03:112-113。
[3] 张致智. 浅谈 $380 / 220 \mathrm{~V}$ 三相四线系统零线断线的危害及重 复接地的重要性 [J]. 青海水力发电, 2002(3):42-43。

[4] 梁福成. 三相四线制供电系统中零线断线的危险和原因分 析及预防措施 [J]. 硅谷, 2013(7):169-170。

[5] 潘涁维. 防范 “断零”与 $\mathrm{PE}$ 线的重复接地 $[\mathrm{J}]$. 内江科技, 2006(02):147+162。

[6] 张玉山. 浅谈中性线断线的防范措施 [J]. 农村运维人员, 2012,04:28。

[7] 陈全兴, 王晓东, 薛鸿鹏. 低压供电系统零线断线故障的 监测与预防[J]. 科技与生活, 2011(21):105-105。

[8] 袁乐崧. $0.4 \mathrm{kV}$ 三相四线运行中零线重复接地的设计与应用 [J]. 电气技术, 2008(1):71-73。

[9] 罗毅.低压配电线路故障中性线断线的危害及防护措施 [J]. 广东科技,2008(02):85-86。

[10] 孔令文.低压供电系统中性线断线的危害和预防措施 [J].浙 江农村机电,2006(01):17。

[11] 刘青.中性线断线产生危害的原因及防范措施 [J].煤炭科 技,2005(03):57-58。

[12] 王宗玉, 李万生.零线断线的危害及防止对策 [J].农村电 工,2002(11):32。

[13] 刘林芝,赵颖涛,张晓峰.中性线断线的危害及其防护措施 [J]. 郑州工业高等专科学校学报,2001(02):23-24.

[14] 罗林开. 零线断线的危害及预防[A]. 重庆市电机工程学会. 重庆市电机工程学会2010年学术会议论文集[C]. 重庆市电 机工程学会:重庆市科学技术协会, 2010:1。

[15] 宋茂智.380/220伏三相四线制线路中零线断线的危害 [J].建 筑电气,1983(01):40-41。 